\title{
Two-color, two-photon imaging at long excitation wavelengths using a diamond Raman laser
}

Johanna Trägårdh, ${ }^{1,{ }^{*}}$ Michelle Murtagh, ${ }^{1,2}$ Gillian Robb, ${ }^{1}$ Maddy Parsons, ${ }^{3}$ Jipeng

Lin, ${ }^{2}$ David J. Spence, ${ }^{2}$ and Gail McConnell, ${ }^{1}$

${ }^{1}$ Centre for Biophotonics, SIPBS, University of Strathclyde, 161 Cathedral Street, Glasgow, G4 ORE, UK

${ }^{2}$ MQ Photonics, Department of Physics and Astronomy, Macquarie University, NSW 2109, Australia

${ }^{3}$ Randall Division of Cell and Molecular Biophysics, King's College London, Guy's Campus, London, SE11UL,UK

${ }^{*}$ Corresponding author:

Johanna Trägårdh

Centre for Biophotonics, SIPBS,

University of Strathclyde,

161 Cathedral Street, Glasgow, G4 ORE, UK

Phone: +44 (0)141 5484645

Fax: +44 (0) 1415484708

johanna.tragardh@strath.ac.uk

Running title: Two-photon imaging using a diamond Raman laser 
Abstract: We demonstrate that the second-Stokes output from a diamond Raman laser, pumped by a femtosecond Ti:Sapphire laser, can be used to efficiently excite red-emitting dyes by twophoton excitation at $1080 \mathrm{~nm}$ and beyond. We image red fluorescent protein (RFP) expressing HeLa cells, as well as dyes such as Texas Red and Mitotracker Red. We demonstrate the potential for simultaneous two-color, two-photon imaging with this laser by using the residual pump beam for excitation of a green-emitting dye. We demonstrate this for the combination of AlexaFluor 488 and AlexaFluor 568. Because the Raman laser extends the wavelength range of the Ti:Sapphire laser, resulting in a laser system tunable $680 \mathrm{~nm}-1200 \mathrm{~nm}$ it can be used for twophoton excitation of a large variety and combination of dyes.

Key words: Nonlinear microscopy; Fluorescence microscopy; Raman lasers; Fluorescent proteins; Two-photon microscopy.

\section{Introduction}

Two-photon excitation of fluorescence allows imaging deep into tissue with minimal photodamage (Zipfel et al., 2003). Extending the excitation wavelength beyond the range of the Ti:Sapphire laser (680-1080 nm) can further reduce the scattering of tissue and thereby increase penetration depth, as shown by (Anderson \& Parrish, 1981; Horton et al., 2013), as well as allow excitation of red-emitting dyes and fluorescent proteins (Drobizhev et al., 2011)., e.g. mCherry, red fluorescent protein (RFP) (Drobizhev et al., 2011), and Alexa 647 (Kobat et al., 2009), and red-emitting calcium indicators. Such red-emitting dyes are increasingly being used in biomedical microscopy since they facilitate fluorescence imaging with multiple labels as well reduce interference from tissue autofluorescence (Drobizhev et al., 2011). 
In this letter we use a diamond Raman laser to extend the wavelength range of a Ti:Sapphire pump laser, and demonstrate its use for two-photon imaging of red-emitting dyes. The Raman shift in diamond is $1332 \mathrm{~cm}^{-1}$, which substantially extends the tuning range of the Ti:Sapphire laser, in particular when using the second-Stokes emission. Such a Raman laser is therefore a means to extend the wavelength range of a type of laser that already exists in many bioimaging facilities. The laser described in this letter allows tuning of the second-Stokes emission to 1200 $\mathrm{nm}$, for a pump wavelength of $900 \mathrm{~nm}$. Femtosecond diamond Raman lasers emitting first Stokes (Lin \& Spence, 2016; Murtagh et al., 2015) and, more recently, second-Stokes (Murtagh et al., 2015b) radiation have been demonstrated, but have, to our knowledge, not been used for imaging. Analogous synchronously-pumped fiber Raman lasers have also recently been developed (Churin et al., 2015).

This setup also generates a two color output, by using the residual pump beam along with the Raman-shifted laser output. Two-color laser systems are attractive for multiple wavelength excitation (because of the spread of peak wavelengths of two-photon absorption cross sections (Drobizhev et al., 2011)) and photo-activation experiments as well for as multimodal imaging (Chu et al., 2001), combining e.g. second or third harmonic generation (SHG, THG) with fluorescence imaging. We demonstrate dual wavelength imaging using the residual pump beam to excite the green-emitting Alexa 488 and the Raman laser to excite the red-emitting Alexa 568. An alternative to a Raman laser for extending the wavelength range of the Ti:Sapphire laser and providing two excitation wavelengths simultaneously, is an optical parametric oscillator (OPO) which, however, if continuous tuning is required is more complicated due to the requirement for phase-matching in the OPO crystal (Linnenbank \& Linden, 2014) and the need for cavity realignment because of the beam walk-off resulting from angle tuning. Cr:Forsterite lasers (Sun et 
al., 2004) provide tunable emission in the range $1220-1270 \mathrm{~nm}$, but no residual pump beam for excitation of green-emitting dyes.

In addition to multi-colour two-photon imaging at long wavelengths using an OPO e.g. (Mahou et al. 2012), multi-colour two-photon excitation has been demonstrated, at shorter wavelengths than used here, using a number of methods such as in (Butko et al., 2011) where two tunable femtosecond lasers were used in parallel and the relative excitation efficiency controlled by polarization. Although very flexible, two fs lasers is a high-cost solution. Tillo et al. (Tillo et al. 2010) demonstrated excitation of multiple fluorescent proteins using only a single laser wavelength, by utilizing transitions to the higher lying singlet states $\left(S_{0^{-}}>S_{n}\right)$ in the redder emitting dye. Although a single excitation wavelength is convenient, it somewhat limits the possible combinations of dyes (by the need to have a large energy separation of the $\left(\mathrm{S}_{0^{-}}>\mathrm{S}_{1}\right)$ transitions) and makes control of the relative excitation efficiency difficult. Similarly, (Yamanaka et al. 2015) used visible wavelengths to simultaneously two-photon excite multiple fluorescent proteins in the UV via $\mathrm{S}_{0^{-}}>\mathrm{S}_{\mathrm{n}}$ transitions. For the Raman laser demonstrated here, the pump and second Stokes wavelengths are necessarily correlated, but tunable and still allows reasonable flexibility in the choice of dyes as well as independent control of the laser intensity. Other methods include phase-shaping the output of a broadband fs laser (Brenner et al. 2013) which is, however, less likely to be available in a bioimaging lab. For a further discussion of methods for multi-colour two-photon excitation see (Brenner et al. 2013).

\section{Materials and Methods}

\section{Sample preparation}


We performed two-photon imaging of HeLa cells expressing RFP-Lifeact. The cells were maintained as previously described (Jayo et al., 2012). The cells were fixed for 20 minutes using 3.7\% paraformaldehyde (Sigma-Aldrich) in PBS and washed twice in PBS. The coverslip was then mounted using Vectashield Hard-Set Mounting Medium (Vector Laboratories).

We also imaged FluoCells prepared slides (Life Technologies) \#1, \#2 and \#3.

\section{Two-photon microscopy}

The setup of the synchronously-pumped Raman laser is shown in Fig. 1. The diamond crystal (Type IIa, CVD-grown, 9 mm-long) had broadband AR-coatings, 1-3\% reflectivity over 796 $1200 \mathrm{~nm}$. The Ti:Sapphire pump laser (Coherent Chameleon Ultra II) generated $200 \mathrm{fs}$ pulses at $840 \mathrm{~nm}$ with a pulse repetition frequency of $80 \mathrm{MHz}$. The pump beam was polarized parallel to the [111] axis of the diamond crystal using a half-wave plate. The pump beam was focused through the input mirror M1 into the center of the diamond crystal, using a $150 \mathrm{~mm}$ focal length lens, to a focal spot radius $\omega_{0}=27 \mu \mathrm{m}$. The spot size was adjusted using a beam expander. The Raman laser was configured as a ring cavity, consisting of two concave mirrors (M1 and M2, radius of curvature $(\mathrm{ROC})=200 \mathrm{~mm}$, separated by approximately $210 \mathrm{~mm}$ ) and two plane mirrors (M3 and M4). The mirror coatings are specified in Fig. 1: the cavity was high-Q for the Stokes wavelengths, and had a $30 \%$ output coupler for the second-Stokes wavelengths. The laser was continuously tuned by tuning the pump laser and adjusting the cavity length by translating mirror M4 to match the round-trip time for each emission wavelength to the pulse period of the pump laser.

With this choice of input mirror, the second-Stokes output could be tuned from $1080 \mathrm{~nm}$ to 1200 $\mathrm{nm}$ for pump wavelengths from $840 \mathrm{~nm}$ to $900 \mathrm{~nm}$ (Fig. 2(a)). The spectra were measured using an optical spectrum analyser (HP 86140A). The second-Stokes wavelength was not always 
exactly two Stokes shifts longer than the pump center wavelength owing to spectral broadening of the Stokes field by self-phase modulation (Murtagh et al., 2015b). The output power of the second-Stokes emission is shown in Fig. 2(b) and was up to $80 \mathrm{~mW}$, making the laser suitable for two-photon imaging of biological specimens. At pump wavelengths above $900 \mathrm{~nm}$ the laser emitted the first Stokes, extending the tuning range on the short wavelength side down to 1035 $\mathrm{nm}$. The pulse durations for the second-Stokes output pulses using pump wavelengths of $840 \mathrm{~nm}$ and $890 \mathrm{~nm}$ were measured to be $1.0 \mathrm{ps}$ using an autocorrelator (APE Carpe) and assuming a Gaussian pulse shape. The detailed dynamics and characteristics of this type of diamond Raman laser is described in (Murtagh et al., 2015b). We note that the bandwidth and output power presented here is much smaller than in (Murtagh et al., 2015b), likely because of a somewhat lower quality diamond crystal. For imaging however, the main concern is the pulse length, which was similar to (Murtagh et al., 2015b).

For the imaging experiments, the Raman laser output was filtered by a $1000 \mathrm{~nm}$ long pass (LP) filter (Thorlabs FEL1000) to remove the pump laser light and coupled into a home built scanning multi-photon microscope. The microscope has been previously described in (Trägårdh et al., 2015). The residual pump laser output was also coupled into the microscope and overlapped with the Raman laser emission using a flip mirror. The light was focused using a Nikon Plan Apo 60X NA1.4 oil immersion objective or a Nikon Fluor S 40X NA1.3 oil immersion objective. The pulse length for the Raman laser was measured to be $1 \mathrm{ps}$ at $1080 \mathrm{~nm}$ at the sample plane, and the pulse length of the residual pump pulse was measured to $230 \mathrm{fs}$. The generated two-photon fluorescence signal was collected in transmission by the condenser lens (NA 0.8) and filtered using a $700 \mathrm{~nm}$ short pass filter (E700SP, Chroma Technology). To detect emission from Alexa 488 excited by the residual pump beam, we further used a 525/39 $\mathrm{nm}$ band pass filter (Semrock, 
Brightline). The signal was detected using a photomultiplier tube (Thorlabs PMM02). The image had 500x500 pixels with a pixel dwell time of $10 \mu$ s and we used a frame average of 4 . For imaging FluoCells slide \#1 and \#2 and for measuring the power dependence of the fluorescence signal, the image had 500x500 pixels with a pixel dwell time of $20 \mu \mathrm{s}$. For comparison, twophoton fluorescence images of RFP expressing cells were also obtained using a commercial OPO at a wavelength of $1080 \mathrm{~nm}$, a pulse duration of $280 \mathrm{fs}$ at the sample plane, a bandwidth of 25 $\mathrm{nm}$ and a repetition frequency of $80 \mathrm{MHz}$ (Coherent Chameleon OPOVis).

\section{Results and Discussion}

Figure 3 shows a two-photon fluorescence image of RFP-Lifeact in a fixed HeLa cell. The RFPLifeact transfection results in specific RFP labelling of F-actin, and for these cells we can clearly see the filopodia in the red detection channel. This image clearly shows that two-photon imaging is possible using the second-Stokes pulses from the diamond Raman laser without pulse compression.

We compared the efficiency of excitation with the Raman laser and the OPO by imaging a fixed RFP expressing cell (data not shown). The signal level for the same time-averaged excitation power was four times higher for excitation with the OPO, as could be expected from the four times shorter pulse length of the OPO. In future work, it would be desirable to generate shorter pulses from the diamond Raman laser; this may be possible using separate cavity length control for the first- and second-Stokes field to produce a compressible second-Stokes output pulse (Murtagh et al., 2015b). 
We confirmed that the contrast in the image was from a two-photon excitation process by acquiring a series of images at different excitation powers. The average slope of a linear fit to the $\log -\log$ plot of the signal for 10 regions of interest across 3 samples was $1.7 \pm 0.1$, confirming the second order process.

Deviations such as this from the quadratic power dependence of the fluorescence are reported and discussed in literature, and commonly attributed to saturation of the dye or photo-bleaching, but also to stimulated emission, excited state absorption, residual one-photon absorption, intersystem crossing (ISC) and electric field induced changes to the dye (Xu and Webb 1996, Xu et al. 1996, Bradley et al. 1972). We do not observe significant photo-bleaching and the excitation powers used here are too low for saturation and electric-field induced changes to the dye (Xu and Webb 1996, Xu et al. 1996). The excitation wavelength (1080 nm) is too long for one-photon absorption and stimulated emission. We therefore tentatively attribute the slope of less than 2 to higher order absorption to states that do not emit fluorescence (possibly involving ISC to a non-fluorescent state) and excited state absorption.

We used the laser for two-colour imaging, exciting Alexa 568 using the Raman laser and Alexa 488 using the residual pump beam (Fig. 4(c)). The image is an overlay of the images acquired using the excitation at $1080 \mathrm{~nm}$ and the residual pump at $840 \mathrm{~nm}$. We also used the output from the Raman laser to successfully excite Texas Red, and Mitotracker Red (Fig. 4) as well as Alexa 594 and ds-Red (data not shown) thereby demonstrating the versatile use of the Raman laser for two-photon excitation of red-emitting dyes. Although the second-Stokes output in our laser could only be tuned between $1080 \mathrm{~nm}$ and $1200 \mathrm{~nm}$, a different choice of mirror coatings would allow other pump wavelengths to suit other dyes, as well as extend the wavelength range further, to match the wavelength range of a Cr:Forsterite laser. 


\section{Conclusions}

In conclusion, we have demonstrated fluorescence imaging by two-photon excitation of redemitting dyes, including RFP, using the second-Stokes output from a diamond Raman laser. We also demonstrated that the combination of the Raman output and the residual pump beam can be used for dual color imaging. The latter is important since there is quite a spread of the peak wavelength of the two-photon absorption cross sections of different dyes, and a single laser wavelength would not suffice for imaging of samples with multiple labels.

\section{Acknowledgments}

This research was supported by the MRC grant MR/K015583/1 and Australian Research Council Linkage Project LP110200545, in association with M Squared Lasers Ltd. M. Murtagh is equally supported by an iMQRES scholarship at Macquarie University and a studentship at University of Strathclyde. The dataset associated with this research is available at https://pure.strath.ac.uk.

\section{References}

ANDERSON, R. R. \& PARRISH, J. A. (1981). The optics of human skin. The Journal of investigative dermatology 77, 13-19.

Bradley, D. J., Hutchinson, M. H. R., Koetser, H., Morrow, G., New, G. H. C., \& Petty M. S. (1972) Interactions of Picosecond Laser Pulses with Organic Molecules. I. Two-Photon Fluorescence Quenching and Singlet States Excitation in Rhodamine Dyes. Proc. R. Soc. London A 328, 97-121.

Brenner, M.H., CAi, D., Swanson, J.A. \& Ogilvie, J. P . (2013) Two-photon imaging of multiple fluorescent proteins by phase-shaping and linear unmixing with a single broadband 
laser. Opt Express. 21, 17256-17264.

Butko, M.T., Drobizhev, M., Makarov, N. S., Rebane A., Brinkman B. C., \& Gleeson J. G. (2011) Simultaneous multiple-excitation multiphoton microscopy yields increased imaging sensitivity and specificity. BMC Biotechnol. 11:20 doi: 10.1186/1472-6750-11-20.

Chu, S. W., Chen, I. H., LiU, T. M., Chen, P. C., Sun, C. K. \& Lin, B. L. (2001). Multimodal nonlinear spectral microscopy based on a femtosecond Cr:forsterite laser. Optics letters 26, $1909-11$.

Churin, D., Olson, J., Norwood, R. A, Peyghambarian, N. \& Kieu, K. (2015). High-power synchronously pumped femtosecond Raman fiber laser. Optics letters 40, 2529-32.

Drobizhev, M., Makarov, N. S., Tillo, S. E., Hughes, T. E. \& Rebane, A. (2011). Twophoton absorption properties of fluorescent proteins. Nature methods 8, 393-399.

Horton, N. G., Wang, K., Kobat, D., Clark, C. G., Wise, F. W., Schaffer, C. B. \& Xu, C. (2013). In vivo three-photon microscopy of subcortical structures within an intact mouse brain. Nature photonics 7, 205-209.

JAYO, A., PARSONS, M. \& ADAMS, J. C. (2012). A novel Rho-dependent pathway that drives interaction of fascin-1 with p-Lin-11/Isl-1/Mec-3 kinase (LIMK) 1/2 to promote fascin1/actin binding and filopodia stability. BMC biology 10, 72 .

Kobat, D., Durst, M. E., Nishimura, N., Wong, A. W., Schaffer, C. B. \& Xu, C. (2009). Deep tissue multiphoton microscopy using longer wavelength excitation. Optics Express 17, 13354.

LiN, J., \& SPENCE, D. J. (2016). 25.5 fs dissipative-soliton diamond Raman laser. Optics Letters, in press (2016).

LiNNENBANK, H. \& LiNDEN, S. (2014). High repetition rate femtosecond double pass optical 
parametric generator with more than $2 \mathrm{~W}$ tunable output in the NIR. Optics express 22, 18072.

Mahou, P., Zimmerley, M., Loulier, K., Matho, K.S., Labroille, G., Morin, X., Supatto, W., Livet, J., DÉBARRE, D. \& BeAurePaire, E. (2012) Multicolor two-photon tissue imaging by wavelength mixing. Nat. Methods $\mathbf{9}, 815-818$.

Murtagh, M., Lin, J., Mildren, R. P., McConnell, G. \& SPEnce, D. J. (2015). Efficient diamond Raman laser generating 65 fs pulses. Optics express 23, 15504-13.

MurTaGH, M., Lin, J., TRÄGÅRDH, J., MCCONNELl, G., \& SPENCE, D. J (2015b). Ultrafast second-Stokes diamond Raman laser. Optics Express, in press (2016).

Sun, C. K., Chu, S. W., Chen, S. Y., Tsai, T. H., LiU, T. M., Lin, C. Y. \& Tsai, H. J. (2004). Higher harmonic generation microscopy for developmental biology. Journal of Structural Biology 147, 19-30.

Tillo S.E., Hughes T. E., Makarov N.S., Rebane A. \& Drobizhev M. (2010) A new approach to dual-color two-photon microscopy with fluorescent proteins. BMC Biotechnol. 10:6 DOI: 10.1186/1472-6750-10-6.

TrÄGÅRdH, J., RobB, G., AMOR, R., Amos, W. B., Dempster, J. \& McCONNELL, G. (2015). Exploration of the two-photon excitation spectrum of fluorescent dyes at wavelengths below the range of the Ti:Sapphire laser. Journal of Microscopy 259, 210-218.

Xu, C., Zipfel W., Shear, J. B., Williams, R. M. \& Webb, W. W. (1996) Multiphoton fluorescence excitation: new spectral windows for biological nonlinear microscopy., Proc. Natl. Acad. Sci. USA. 93, 10763-10768.

Xu, C. \& WeBB, W. W. (1996) Measurement of two-photon excitation cross sections of molecular fluorophores with data from 690 to $1050 \mathrm{~nm}$. J. Opt. Soc. Am. B 13, 481-491. 
Yamanaka, M., Saito, K., Smith, N. I., Arai, Y., Uegaki, K., Yonemaru, Y., Mochizuki, K., KaWATA S., NAGAI, T. \& FuJiTA, K. (2015) Visible-wavelength two-photon excitation microscopy for fluorescent protein imaging. J. Biomed. Opt. 20, 101202-1-11.

ZIPFEL, W. R., Williams, R. M. \& WEBB, W. W. (2003). Nonlinear magic: multiphoton microscopy in the biosciences. Nature biotechnology 21, 1369-1377. 


\section{Figure Captions}

Fig 1: (a) Layout of the Raman laser. 1/2: half-wave plate, BE: beam expander for mode matching, L1: focusing lens, M1: ROC = 200 mm, 1.5\%R @ 840-900nm, HR 940-1300nm, M2: ROC = 200 mm, HR @ 840-900nm, HR 940-1300nm M3: plane mirror, HR @ 940-1300 nm, M4: plane mirror, output coupler, HR 940-1030nm, T=30\% @ 1040-1300nm, D: diamond.

Fig 2: (a) Raman laser emission spectra for pump wavelengths from $840 \mathrm{~nm}$ to $900 \mathrm{~nm}$. The calculated positions of the second-Stokes wavelengths are indicated in the figure (red lines and crosses). (b) Raman laser output and pump power.

Fig 3: False color image of an RFP-Lifeact expressing HeLa cell. The excitation wavelength is $1080 \mathrm{~nm}(11 \mathrm{~mW})$ and we used a $40 \mathrm{X}$ NA1.3 objective lens. Scalebar is $25 \mu \mathrm{m}$.

Fig 4: False color image of (a) BPAE cells with the F-actin labelled with Texas Red (FluoCells slide \#2). Excitation wavelength: $1100 \mathrm{~nm}(13 \mathrm{~mW}), 40 X$ NA1.3 objective lens. (b) BPAE cells with the mitochondria labelled with Mitotracker Red CMXRos (FluoCells slide \#1). Excitation wavelength: $1130 \mathrm{~nm}(16 \mathrm{~mW}), 60 X$ NA1.4 objective lens. (c) Cryostat section of mouse kidney stained with Alexa Fluor 488 (green) and Alexa Fluor 568 (red) (FluoCells slide \#3). The excitation wavelengths are $1080 \mathrm{~nm}(13 \mathrm{~mW})$ and $840 \mathrm{~nm}(11 \mathrm{~mW}), 60 X$ NA1.4 objective lens. Scalebars are $50 \mu \mathrm{m}$. 